\title{
Small Label Classes in 2-Distinguishing Labelings
}

\author{
Debra L. Boutin \\ Hamilton College, Clinton, NY 13323, USA
}

Received 11 September 2007, accepted 25 November 2008, published online 29 November 2008

\begin{abstract}
A graph $G$ is said to be 2-distinguishable if there is a labeling of the vertices with two labels so that only the trivial automorphism preserves the labels. Call the minimum size of a label class in such a labeling of $G$ the cost of 2-distinguishing $G$ and denote it by $\rho(G)$. This paper shows that for $n \geq 5,\left\lceil\log _{2} n\right\rceil+1 \leq \rho\left(Q_{n}\right) \leq 2\left\lceil\log _{2} n\right\rceil-1$, where $Q_{n}$ is the hypercube of dimension $n$.
\end{abstract}

Keywords: Graph, distinguishing labeling, automorphism group.

Math. Subj. Class.: 05C15, 05C25

\section{Introduction}

A labeling of the vertices of a graph $G$ with the integers $1, \ldots, d$ is called a $d$-distinguishing labeling if no nontrivial automorphism of $G$ preserves the labels. A graph is called $d$ distinguishable if it has a $d$-distinguishing labeling. Albertson and Collins introduced distinguishing in [2]. Recent work shows that large members of many infinite families of graphs are 2-distinguishable. These graph families include hypercubes $Q_{n}$ with $n \geq 4$ [3], nontrivial Cartesian powers of a connected graph $G \neq K_{2}, K_{3}$ [8], Kneser graphs $K_{n: k}$ with $n \geq 6, k \geq 2$ [1], and (with seven small exceptions) 3-connected planar graphs [6]. Recently Wilfried Imrich posed the following question: "What is the minimum number of vertices in a label class of a 2-distinguishing labeling for the hypercube $Q_{n}$ ?" This question can be extended to any family of 2-distinguishable graphs.

Let $G$ be a 2-distinguishable graph. Call a label class in a 2-distinguishing labeling of $G$ a distinguishing class. Call the minimum size of such a class in $G$ the cost of 2-distinguishing $G$ and denote it by $\rho(G)$. The labeling provided for $Q_{n}$ by Bogstad and Cowan in [3] shows that for $n \geq 4, \rho\left(Q_{n}\right) \leq n+2$. The best result known when Imrich posed the question mentioned above was $\rho\left(Q_{n}\right) \approx \sqrt{n}$ [7]. For $n \geq 5$, this paper shows that $\rho\left(Q_{n}\right) \leq 2\left\lceil\log _{2} n\right\rceil-1$ and that this is within a factor of two of a natural lower bound (discussed below). To give a sense

E-mail address: dboutin@hamilton.edu (Debra L. Boutin) 
of this result, note that though $Q_{16}$ has $2^{16}$ vertices and $16 ! \times 2^{16}$ automorphisms, it can be distinguished, effectively eliminating all symmetry, by coloring just seven vertices red and all others blue.

A significant tool used in this work is the determining set [4], a set of vertices whose pointwise stabilizer is trivial. Albertson and Boutin showed that a graph is $d$-distinguishable if and only if it has a determining set that is $(d-1)$-distinguishable [1]. When $d=2$ this translates as: a graph is 2-distinguishable if and only if it has a determining set with the property that any automorphism that preserves the set must fix it pointwise. This shows that $\rho(G)$ is bounded below by the size of a smallest determining set for $G$. For $Q_{n}$ this bound is $\left\lceil\log _{2} n\right\rceil+1$ [5]. We will use the connection between determining sets and distinguishing labelings, along with particular determining sets found in [5], to create a distinguishing class for $Q_{n}$ that is smaller than twice this lower bound.

The paper is organized as follows. Definitions and facts about determining sets, Cartesian products, and distinguishing labelings are given in Section 2. This section also sets out the key idea tying together determining sets and distinguishing labelings. For $n \geq 5$, Section 3 gives a set of $2\left\lceil\log _{2} n\right\rceil-1$ vertices of $Q_{n}$ and proves that it is a distinguishing class. Section 4 lists some open questions.

\section{Background}

\subsection{Determining Sets}

Let $G$ be a graph. A subset $U \subseteq V(G)$ is said to be a determining set for $G$ if whenever $g, h \in \operatorname{Aut}(G)$ and $g(x)=h(x)$ for all $x \in U$, then $g=h$. Thus every automorphism of $G$ is uniquely determined by its action on a determining set. Every graph has a determining set since any set containing all but one vertex is determining. There are graphs, e.g. $K_{n}$ and $K_{1, n}$, for which such a determining set is optimal or within one of being optimal. The determining number of $G$, denoted here by $\operatorname{Det}(G)$, is the minimum $r$ such that $G$ has a determining set of cardinality $r$.

Recall that the set stabilizer of $U \subseteq V(G)$ is the set of all $\phi \in \operatorname{Aut}(G)$ for which $\phi(x) \in U$ for all $x \in U$. In this case we say that $\phi(U)=U$. The pointwise stabilizer of $U$ is the set of all $\phi \in \operatorname{Aut}(G)$ for which $\phi(x)=x$ for all $x \in U$. It is easy to see that $U \subseteq V(G)$ is a determining set for $G$ if and only if the pointwise stabilizer of $U$ is trivial.

\subsection{Cartesian Products}

Recall that the Cartesian product of graphs $G$ and $H$, denoted by $G \square H$, has vertex set $V(G) \times V(H)$ with an edge between vertices $(x, u)$ and $(y, v)$ if either $x$ is adjacent to $y$ in $G$ and $u=v$, or $u$ is adjacent to $v$ in $H$ and $x=y$. The Cartesian power $H^{k}$ is the Cartesian product of $H$ with itself $k$ times.

A good reference for Cartesian products is [9]. Recall that $H$ is prime with respect to the Cartesian product if it cannot be written as the Cartesian product of two smaller graphs. Further, every connected graph can be written uniquely (up to order) as the Cartesian product of prime factors, $G=G_{1} \square \cdots \square G_{m}$.

Let $U=\left\{V_{1}, \ldots, V_{r}\right\}$ be an ordered subset of vertices of $G=G_{1} \square \cdots \square G_{m}$. Let $M=M_{U}$ be the $r \times m$ matrix whose $i^{t h}$ row contains the coordinates for $V_{i}$ with respect 
to the prime factor decomposition of $G$. Call this the characteristic matrix for $U$. Note that the $j^{t h}$ column of $M$ consists of the $j^{t h}$ coordinates of $V_{1}, \ldots, V_{r}$ and can be denoted $\left[\begin{array}{lll}V_{1, j} & \ldots & V_{r, j}\end{array}\right]^{T}$. We say the $j^{t h}$ and $k^{t h}$ columns of $M,\left[V_{1, j} \ldots V_{r, j}\right]^{T}$ and $\left[V_{1, k} \ldots V_{r, k}\right]^{T}$, are isomorphic images of each other if there exists an isomorphism $\varphi: G_{j} \rightarrow G_{k}$ so that $\varphi\left(V_{i, j}\right)=V_{i, k}$ for all $i$. These definitions allow us to state criteria for a set to be a determining set as follows.

Lemma 1. [5] Let $G$ be a connected graph and $G=G_{1} \square \cdots \square G_{m}$ the prime factor decomposition for $G$ with respect to the Cartesian product. A set of vertices $U$ is a determining set for $G$ if and only if each column of the characteristic matrix $M$ for $U$ contains a determining set for the appropriate factor of $G$ and no two columns of $M$ are isomorphic images of each other.

\subsection{Distinguishing Labelings}

A labeling $f: V(G) \rightarrow\{1, \ldots, d\}$ is said to be $d$-distinguishing if $\phi \in \operatorname{Aut}(G)$ and $f(\phi(x))=f(x)$ for all $x \in V(G)$ implies that $\phi=i d$. Every graph has a distinguishing labeling since each vertex can be assigned a distinct label. Furthermore, there are graphs, e.g. $K_{n}$ and $K_{1, n}$, for which such a labeling is optimal or within one of being optimal. A graph is called $d$-distinguishable if it has a $d$-distinguishing labeling.

We will also need to know what it means for a subset of vertices to be $d$-distinguishable. Let $U \subseteq V(G)$. A labeling $f: U \rightarrow\{1, \ldots, d\}$ is called $d$-distinguishing if whenever $\phi \in \operatorname{Aut}(G)$ so that $\phi(U)=U$ and $f(\phi(x))=f(x)$ for all $x \in U$ then $\phi(x)=x$ for all $x \in U$. Note that though such a $\phi$ fixes $U$ pointwise, it is not necessarily trivial; it may permute vertices in the complement of $U$. Then by definition, $U$ is 1-distinguishable if every automorphism that preserves $U$ fixes it pointwise.

The following theorem ties together determining sets and distinguishing labelings and facilitates the work in this paper.

Theorem 2. [1] A graph is $d$-distinguishable if and only if it has a determining set that is $(d-1)$-distinguishable.

In particular, suppose $U$ is a 1-distinguishable determining set. The fact that it is 1 distinguishable means that any automorphism that preserves $U$ as a set also fixes it pointwise. The fact that it is a determining set means that the only automorphism that fixes it pointwise is the trivial automorphism. Thus if we label the vertices of $U$ with ones and the vertices of its complement with twos, only the trivial automorphism preserves the label classes. Therefore $U$ is a distinguishing class of a 2-distinguishing labeling. Thus to find a distinguishing class, we will look for a (small) determining set for which no nontrivial automorphism both preserves the set and permutes vertices within the set. One can think of such a set as having no "internal symmetry."

\section{The Hypercubes}

Recall that the $n$-cube, or hypercube of dimension $n$, is the Cartesian product of $K_{2}$ with itself $n$ times. That is, $Q_{n}=K_{2}^{n}$. Thus we can represent the vertices of $Q_{n}$ as strings of $n$ zeros and ones. For $n \geq 5$ we will construct a distinguishing class of size $2\left\lceil\log _{2} n\right\rceil-1$ for $Q_{n}$. We will start by defining a set $U_{r} \subseteq V\left(Q_{2^{r}}\right)$ for $r \geq 3$. In Theorem 5 we will show that 
$U_{r}$ is indeed a distinguishing class for $Q_{2^{r}}$. In Theorem 6 we will show that if $r=\left\lceil\log _{2} n\right\rceil$ the same is true for the projection of $U_{r}$ into $Q_{n}$ obtained by projecting each vertex onto its first $n$ coordinates.

First let us define $U_{r}$. For $0 \leq k \leq r$, we call each of the $2^{r-k}$ consecutive sequences of $2^{k}$ coordinates in a vertex of $Q_{2^{r}}$ a block of length $2^{k}$. For $i=1, \ldots, r$, let $V_{i}$ be the vertex of $Q_{2^{r}}$ consisting of blocks of $2^{i-1}$ ones alternating with blocks of $2^{i-1}$ zeros. Let $V_{0}$ be the vertex with one in its second coordinate and zeros in all others. For $i=1, \ldots, r-2$, let $X_{i}$ be the vertex of $Q_{2^{r}}$ that agrees with $V_{i}$ and $V_{i+1}$ on the coordinates in which they agree and that has a one in every other coordinate. We can think of $X_{i}$ as the "OR" of $V_{i}$ and $V_{i+1}$; it has a one in a coordinate if either $V_{i}$ or $V_{i+1}$ has a one there, and a zero otherwise. Alternately, $X_{i}$ can be described as having the repeating pattern: three blocks of $2^{i-1}$ ones followed by one block of $2^{i-1}$ zeros. Let $U=U_{r}=\left\{V_{0}, \ldots, V_{r}, X_{1}, \ldots, X_{r-2}\right\}$

Example 3. $U_{4}$ contains the following vertices.

$$
\begin{aligned}
& V_{0}=0100000000000000 \\
& V_{1}=1010101010101010 \\
& V_{2}=1100110011001100 \\
& V_{3}=1111000011110000 \\
& V_{4}=1111111100000000 \\
& X_{1}=1110111011101110 \\
& X_{2}=1111110011111100
\end{aligned}
$$

The proofs of Theorems 5 and 6 make extensive use of distances between elements of $U$. Due to the repeating nature of the coordinates of our vertices, these distances are reasonable to compute. The details are given below and are summarized in Table 1.

Consider $d\left(V_{i}, V_{j}\right)$ where $1 \leq i<j \leq r$. Each block of length $2^{j-1}$ in $V_{j}$ (which contains only zeros or only ones) corresponds to $2^{j-i}$ blocks of length $2^{i-1}$ in $V_{i}$ (which alternate between ones blocks and zeros blocks). Thus $V_{i}$ and $V_{j}$ disagree in half their coordinates. Therefore $d\left(V_{i}, V_{j}\right)=2^{r-1}$ for $1 \leq i<j \leq r$.

Consider $d\left(X_{i}, V_{i}\right)$ and $d\left(X_{i}, V_{i+1}\right)$ where $1 \leq i \leq r-2$. Note that since $i \geq 1$, precisely half the coordinates of $V_{i}$ that disagree with $V_{i+1}$ are zeros and thus disagree with $X_{i}$ also. The same statement holds for $V_{i+1}$. Thus $d\left(X_{i}, V_{i}\right)=d\left(X_{i}, V_{i+1}\right)=\frac{1}{2} d\left(V_{i}, V_{i+1}\right)=2^{r-2}$ for $1 \leq i \leq r-2$.

Consider $d\left(X_{i}, X_{i+1}\right)$ where $1 \leq i \leq r-3$. There are eight blocks of length $2^{i-1}$ in $X_{i}$ to every four blocks of length $2^{i}$ of $X_{i+1}$. By comparing the repeating patterns of these blocks we see that $X_{i}$ and $X_{i+1}$ disagree on the $4^{t h}$ and $7^{\text {th }}$ of these eight blocks. Thus $d\left(X_{i}, X_{i+1}\right)=\frac{2}{8} 2^{r}=2^{r-2}$ for $1 \leq i \leq r-3$.

Consider $d\left(X_{i}, X_{j}\right)$ where $1 \leq i \leq j-2 \leq r-4$. Since $i \leq j-2$, an integer multiple of four blocks of $X_{i}$ correspond to a single block of zeros or ones in $X_{j}$. The four block pattern of $X_{i}$ disagrees $\frac{1}{4}$ of the time with a ones block of $X_{j}$ and $\frac{3}{4}$ of the time with a zeros block of $X_{j}$. Since $\frac{3}{4}$ of the blocks of $X_{j}$ are ones and $\frac{1}{4}$ are zeros, we get that $X_{i}$ and $X_{j}$ disagree $\frac{3}{4} \cdot \frac{1}{4}+\frac{1}{4} \cdot \frac{3}{4}=\frac{3}{8}$ of the time. Thus $d\left(X_{i}, X_{j}\right)=3 \cdot 2^{r-3}$ for $1 \leq i \leq j-2 \leq r-4$.

Consider $d\left(X_{i}, V_{j}\right)$ where $1 \leq i \leq j-2 \leq r-2$. Again since $i \leq j-2$, an integer multiple of four blocks of $X_{i}$ corresponds to a single block of $V_{j}$. The four block pattern of 
$X_{i}$ disagrees with ones blocks and zeros blocks as described above. However, $V_{j}$ has half its blocks ones and half zeros. Thus $X_{i}$ and $V_{j}$ disagree $\frac{1}{2} \cdot \frac{1}{4}+\frac{1}{2} \cdot \frac{3}{4}=\frac{1}{2}$ the time. Thus $d\left(X_{i}, V_{j}\right)=\frac{1}{2} \cdot 2^{r}=2^{r-1}$ for $1 \leq i \leq j-2 \leq r-2$.

Consider $d\left(V_{i}, X_{j}\right)$ where $1 \leq i \leq j-1 \leq r-3$. Since $i \leq j-1$, an integer multiple of two blocks of $V_{i}$ correspond to a single block of $X_{j}$. Such a pair of blocks disagrees with a single block of $X_{j}$ precisely half the time. Thus $d\left(X_{i}, V_{j}\right)=2^{r-1}$ for $1 \leq i \leq j-1 \leq r-3$.

Consider $d\left(V_{0}, V_{i}\right)$ where $1 \leq i \leq r$. Since for $i \geq 1, V_{i}$ has exactly half of its coordinates zero, if $V_{0}$ consisted of all zeros we would have $d\left(V_{0}, V_{i}\right)=2^{r-1}$. However since the second coordinate of $V_{0}$ is one, which disagrees with the second coordinate of $V_{1}$ and agrees with all other $V_{i}$ we have that $d\left(V_{0}, V_{1}\right)=2^{r-1}+1$, and $d\left(V_{0}, V_{i}\right)=2^{r-1}-1$ for $2 \leq i \leq r$.

Consider $d\left(V_{0}, X_{i}\right)$ where $1 \leq i \leq r-2$. Because $X_{i}$ has three blocks of ones followed by one block of zeros, $X_{i}$ has three quarters of its coordinates ones. Adjusting for the one in the second coordinate of $V_{0}$ yields $d\left(V_{0}, X_{i}\right)=\frac{3}{4} 2^{r}-1=3 \cdot 2^{r-2}-1$ for $1 \leq i \leq r-2$.

Note that the only pairs of vertices of $U$ at distance $2^{r-2}$ are $\left\{X_{i}, V_{i}\right\},\left\{X_{i}, V_{i+1}\right\}$, and $\left\{X_{i}, X_{i+1}\right\}$. We will call these related pairs because they have the distance relationship we will use to show that $U$ is 1 -distinguishable. Other pairs of vertices in $U$ are called non-related pairs.

\section{Related Pairs}

$\begin{array}{lll}d\left(X_{i}, V_{i}\right) & 2^{r-2} & \text { for } 1 \leq i \leq r-2 \\ d\left(X_{i}, V_{i+1}\right) & 2^{r-2} & \text { for } 1 \leq i \leq r-2 \\ d\left(X_{i}, X_{i+1}\right) & 2^{r-2} & \text { for } 1 \leq i \leq r-3\end{array}$

\section{Non-Related Pairs}

$\begin{array}{llc}d\left(V_{0}, X_{i}\right) & 3 \cdot 2^{r-2}-1 & \text { for } 1 \leq i \leq r-2 \\ d\left(V_{0}, V_{1}\right) & 2^{r-1}+1 & \\ d\left(V_{i}, V_{j}\right) & 2^{r-1} & \text { for } 1 \leq i<j \leq r \\ d\left(X_{i}, V_{j}\right) & 2^{r-1} & \text { for } 1 \leq i \leq r-2,1 \leq j \leq r, \\ & & j \neq i, i+1 \\ d\left(V_{0}, V_{i}\right) & 2^{r-1}-1 & \text { for } 2 \leq i \leq r \\ d\left(X_{i}, X_{j}\right) & 3 \cdot 2^{r-3} & \text { for } 1 \leq i \leq j-2 \leq r-4\end{array}$

Table 1: Distances in $Q_{2^{r}}$

Before stating and proving Theorem 5, we will prove that $U$ is a determining set for $Q_{2^{r}}$. By Lemma 1, $U$ is a determining set for $Q_{2^{r}}$ if and only if each column of the characteristic matrix for $U$ contains a determining set for its associated prime factor and no two columns are isomorphic images of each other. Since any single vertex is a determining set for $K_{2}$, here we need only show that no two columns of $M_{U}$ are isomorphic images of each other.

Let $M_{U}$ be the characteristic matrix for $U=\left\{V_{0}, \ldots, V_{r}, X_{1}, \ldots, X_{r-2}\right\}$ and $M_{T}$ the 
characteristic matrix for $T=\left\{V_{0}, \ldots, V_{r}\right\}$. Note that the matrix $M_{T}$ is given by the first $r+1$ rows of $M_{U}$. See Example 4 for $M_{U}$ in the case $r=4$.

Example 4. The characteristic matrix for $U_{4}$.

$$
\begin{aligned}
& V_{0} \\
& V_{1} \\
& V_{2} \\
& V_{3} \\
& V_{4} \\
& X_{1} \\
& X_{2}
\end{aligned}\left[\begin{array}{llllllllllllllll}
0 & 1 & 0 & 0 & 0 & 0 & 0 & 0 & 0 & 0 & 0 & 0 & 0 & 0 & 0 & 0 \\
1 & 0 & 1 & 0 & 1 & 0 & 1 & 0 & 1 & 0 & 1 & 0 & 1 & 0 & 1 & 0 \\
1 & 1 & 0 & 0 & 1 & 1 & 0 & 0 & 1 & 1 & 0 & 0 & 1 & 1 & 0 & 0 \\
1 & 1 & 1 & 1 & 0 & 0 & 0 & 0 & 1 & 1 & 1 & 1 & 0 & 0 & 0 & 0 \\
1 & 1 & 1 & 1 & 1 & 1 & 1 & 1 & 0 & 0 & 0 & 0 & 0 & 0 & 0 & 0 \\
1 & 1 & 1 & 1 & 1 & 1 & 1 & 0 & 1 & 1 & 1 & 0 & 1 & 1 & 1 & 0 \\
\end{array}\right.
$$

By the proof of [5, Theorem 3], if we take $T$ and replace $V_{0}$ with the vertex of all zeros, we get a determining set for $Q_{2^{r}}$. This means that if we change the entry in the first row second column of $M_{T}$ to a zero, we would have a characteristic matrix in which no pair of columns are isomorphic images of each other. Thus in $M_{T}$ the only pairs of columns that might be isomorphic images of each other are pairs involving the second column. Note that the second column, $\left[\begin{array}{llllll}1 & 0 & 1 & 1 & \cdots & 1\end{array}\right]^{T}$, and the $\left(2^{r}-1\right)^{s t}$ column, $\left[\begin{array}{llllll}0 & 1 & 0 & 0 & \cdots & 0\end{array}\right]^{T}$, are isomorphic images of each other under the isomorphism $(01)$ of $K_{2}$. (Thus $T$ is not a determining set.) If there was another column of $M_{T}$ isomorphic to the second, it would also be isomorphic to the $\left(2^{r}-1\right)^{s t}$, which we argued cannot happen. Thus the second and $\left(2^{r}-1\right)^{s t}$ columns are the only pair of columns of $M_{T}$ that are isomorphic images of each other.

Since the first $r+1$ rows of $M_{U}$ form $M_{T}$, if two columns of $M_{U}$ are isomorphic images of each other, then so are the corresponding columns in $M_{T}$. Thus we only need to check the second and $\left(2^{r}-1\right)^{s t}$ columns of $M_{U}$. We have seen that the first $r+1$ entries in the second and $\left(2^{r}-1\right)^{s t}$ of these columns are isomorphic images of each other under the isomorphism (0 1). However, since $r \geq 3, X_{1}$ exists, provides the $(r+2)^{n d}$ row of $M_{U}$, and has one in each of its second and $\left(2^{r}-1\right)^{s t}$ coordinates. Thus the isomorphism does not continue to the $(r+2)^{n d}$ entries of these columns. Thus no two columns of $M_{U}$ are isomorphic images of each other and therefore $U$ is a determining set for $Q_{2^{r}}$.

We now have the machinery in place to prove that $U_{r}$ is a distinguishing class for $Q_{2^{r}}$.

Theorem 5. If $r \geq 3$, then $Q_{2^{r}}$ has a distinguishing class of size $2 r-1$.

Proof. Define $U=U_{r} \subset Q_{2^{r}}$ as above. Define the distance relationship graph on $U$, denoted $G_{U}$, to be the graph with vertex set $U$ and with edges between two vertices if their distance in $Q_{2^{r}}$ is $2^{r-2}$. The related pairs, $\left\{X_{i}, V_{i}\right\},\left\{X_{i}, V_{i+1}\right\}$, and $\left\{X_{i}, X_{i+1}\right\}$, are precisely those that are adjacent in the distance relationship graph. In particular, in this distance relationship graph $V_{0}$ and $V_{r}$ are isolated vertices, $V_{1}$ and $V_{r-1}$ have degree one, $V_{2}, \ldots, V_{r-2}$ have degree two, $X_{1}, \ldots, X_{r-2}$ have degree greater than two, and there is a connected component induced by $V_{1}, \ldots, V_{r-1}, X_{1}, \ldots, X_{r-2}$ that has $\mathbb{Z}_{2}$ symmetry. See Figure 1 for an illustration when $r=6$.

Since automorphisms necessarily preserve distance, any automorphism in the set stabilizer of $U$ induces an automorphism of the distance relationship graph. But the only nontrivial actions on $G_{U}$ either transpose $V_{0}$ and $V_{r}$, or reflect the nontrivial connected component and therefore transpose $V_{1}$ and $V_{r-1}$, or both. Thus any automorphism that preserves $U$ setwise either transposes $V_{0}$ and $V_{r}$, or $V_{1}$ and $V_{r-1}$, (or both). 


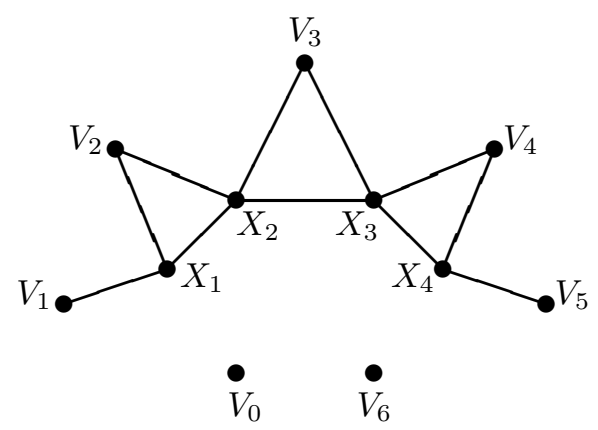

Figure 1: Distance Relationship Graph for $U_{6}$

However, for any $i, 1 \leq i \leq r-2, d\left(V_{0}, X_{i}\right)=3 \cdot 2^{r-2}-1$ while the distance between $V_{r}$ and any vertex of $U-\left\{V_{0}, V_{r}\right\}$ is $2^{r-1}$. Thus $V_{0}$ achieves distances with vertices of $U-\left\{V_{0}, V_{r}\right\}$ that are strictly greater than those achieved by $V_{r}$. Since automorphisms preserve distance, no automorphism that preserves $U$ can transpose $V_{0}$ and $V_{r}$. Thus $V_{0}$ is distinguished from $V_{r}$ within $U$. Further, $d\left(V_{0}, V_{1}\right)=2^{r-1}+1$ and $d\left(V_{0}, V_{r-1}\right)=2^{r-1}-1$. Again, since automorphisms preserve distance, no automorphism in the set stabilizer of $U$ can transpose $V_{1}$ and $V_{r-1}$. Thus $V_{1}$ and $V_{r-1}$ are distinguished within $U$.

Thus any automorphism that preserves $U$ must fix $U$ pointwise. Thus $U$ is 1 -distinguishable. Recall that before beginning Theorem 5 we proved that $U$ is a determining set. Thus $U$ is a 1-distinguishable determining set, i.e. a distinguishing class, of size $2 r-1$ for $Q_{2^{r}}$.

If $2^{r-1}<n<2^{r}$ we will find the desired distinguishing class by projecting $U_{r}$ into $Q_{n}$. The proof that the result is a distinguishing class for $Q_{n}$ is similar to, but more complex than, the proof for $Q_{2^{r}}$.

Theorem 6. If $n \geq 5$, then $Q_{n}$ has a distinguishing class of size $2\left\lceil\log _{2} n\right\rceil-1$.

Proof. By Theorem 5, we get the desired result when $n=2^{r}$ for $r \geq 3$. For $n \geq 5$ and not a power of two, there is some $r \geq 3$ for which $2^{r-1}<n<2^{r}$. For such an $n$, let $p_{n}$ be the projection of $Q_{2^{r}}$ onto $Q_{n}$ by projecting each vertex onto its first $n$ coordinates. Let $U=U_{r}$. Here our goal is to show that $p_{n}(U)$ is a 1-distinguishable determining set for $Q_{n}$. Note that the characteristic matrix for $p_{n}(U)$ is formed from the first $n$ columns of the characteristic matrix for $U$. Since we showed that no two columns in $M_{U}$ are isomorphic images of each other, no two columns in $M_{p_{n}(U)}$ are isomorphic images of each other. Thus $p_{n}(U)$ is a determining set for $Q_{n}$.

Consider the possible distances between vertex pairs in $Q_{n}$. When we project vertices, say $X$ and $Y$, from $Q_{2^{r}}$ into $Q_{n}$ (or from $Q_{n}$ into $Q_{2^{r-1}}$ ) by dropping the appropriate number of rightmost coordinates, we are dropping coordinates in which these vertices may disagree. Thus the distances between projected vertices can only get smaller. In particular the projection of each related pair of $U$ has distance less than or equal to $2^{r-2}$ in $Q_{n}$. Moreover, we conclude that the distance $d\left(p_{n}(X), p_{n}(Y)\right)$ falls between the distance $d\left(p_{2^{r-1}}(X), p_{2^{r-1}}(Y)\right)$ and the distance $d(X, Y)$ (in $Q_{2^{r}}$ ). Again distances in $Q_{2^{r-1}}$ are not hard to find due to the repeating nature of the coordinates of our vertices. They are contained in Table 2. 


\section{Projection of Related Pairs}

$\begin{array}{lll}d\left(p_{n}\left(X_{i}\right), p_{n}\left(V_{i}\right)\right) & 2^{r-3} & \text { for } 1 \leq i \leq r-2 \\ d\left(p_{n}\left(X_{i}\right), p_{n}\left(V_{i+1}\right)\right) & 2^{r-3} & \text { for } 1 \leq i \leq r-2 \\ d\left(p_{n}\left(X_{i}\right), p_{n}\left(X_{i+1}\right)\right) & 2^{r-3} & \text { for } 1 \leq i \leq r-3\end{array}$

\section{Projection of Non-Related Pairs}

$\begin{array}{llc}d\left(p_{n}\left(V_{0}\right), p_{n}\left(V_{r}\right)\right) & 2^{r-1}-1 & \\ d\left(p_{n}\left(V_{0}\right), p_{n}\left(X_{i}\right)\right) & 3 \cdot 2^{r-3}-1 & \text { for } 1 \leq i \leq r-2 \\ d\left(p_{n}\left(V_{0}\right), p_{n}\left(V_{1}\right)\right) & 2^{r-2}+1 & \\ d\left(p_{n}\left(V_{i}\right), p_{n}\left(V_{j}\right)\right) & 2^{r-2} & \text { for } 1 \leq i<j \leq r \\ d\left(p_{n}\left(X_{i}\right), p_{n}\left(V_{j}\right)\right) & 2^{r-2} & \text { for } 1 \leq i \leq r-2, \\ & & 1 \leq j \leq r-1, \\ & & j \neq i, i+1 \\ d\left(p_{n}\left(V_{0}\right), p_{n}\left(V_{i}\right)\right) & 2^{r-2}-1 & \text { for } 2 \leq i \leq r-1 \\ d\left(p_{n}\left(X_{i}\right), p_{n}\left(X_{j}\right)\right) & 3 \cdot 2^{r-4} & \text { for } 1 \leq i \leq j-2 \leq r-4 \\ d\left(p_{n}\left(V_{r}\right), p_{n}\left(X_{i}\right)\right) & 2^{r-3} & \text { for } 1 \leq i \leq r-2\end{array}$

Table 2: Distances of projections into $Q_{n}$, where $n=2^{r-1}$

Suppose that the projection of some related pair has distance $2^{r-2}$ in $Q_{n}$. Since this was also their distance in $Q_{2^{r}}$ this means that the original pair agreed in each of their final $2^{r}-n$ coordinates. Examining the final blocks of the related pairs we find that the maximum agreement is $2^{r-2}$ coordinates and occurs only for $\left\{X_{r-2}, V_{r-2}\right\}$. In particular if $n<3 \cdot 2^{r-2}$ then every related pair is at distance less than $2^{r-2}$.

We will break this proof into two cases: Case 1: $3 \cdot 2^{r-2} \leq n<2^{r}$ and Case 2: $2^{r-1}<$ $n<3 \cdot 2^{r-2}$. In each case we will define a distance relationship graph on the vertex set $p_{n}(U)$ similar to the one defined in the proof of Theorem 5. In Case 1 the distance relationship will be "less than or equal to $2^{r-2}$ " while in Case 2 the distance relationship will be "strictly less than $2^{r-2}$." These definitions will ensure that related pairs will be adjacent in each distance relationship graph.

By Table 2, the non-related vertices that cannot possibly have distance less than or equal to $2^{r-2}$ in $Q_{n}$ where $2^{r-1}<n<2^{r}$ are the pairs $\left\{p_{n}\left(V_{0}\right), p_{n}\left(V_{r}\right)\right\},\left\{p_{n}\left(V_{0}\right), p_{n}\left(X_{i}\right)\right\}$ where $1 \leq i \leq r-2$, and $\left\{p_{n}\left(V_{0}\right), p_{n}\left(V_{1}\right)\right\}$. We will analyze the distances between the projections of all other non-related pairs using their distances when projected into $Q_{3 \cdot 2^{r-2}}$. These are given in Table 3.

Case 1: $3 \cdot 2^{r-2} \leq n<2^{r}$.

When $3 \cdot 2^{r-2} \leq n<2^{r}$, define the distance relationship graph $G_{p_{n}(U)}$ on $p_{n}(U)$ so that there is an edge between a pair of vertices when their distance is less than or equal to $2^{r-2}$. Since all related pairs fit this distance criterion, $G_{p_{n}(U)}$ contains $G_{U}$ (from the proof of Theorem 5) as a subgraph. The distance information from Table 3 allows us to conclude that since $n \geq 3 \cdot 2^{r-2}$ the only edges that might be in $G_{p_{n}(U)}$ but are not in $G_{U}$ would be 


\section{Projection of Some Non-Related Pairs}

$\begin{array}{llc}d\left(p_{n}\left(V_{r-1}\right), p_{n}\left(V_{r}\right)\right) & 2^{r-1} & \\ d\left(p_{n}\left(V_{0}\right), p_{n}\left(V_{r-1}\right)\right) & 2^{r-1}-1 & \\ d\left(p_{n}\left(V_{i}\right), p_{n}\left(V_{j}\right)\right) & 3 \cdot 2^{r-3} & \text { for } 1 \leq i<j \leq r, i \neq r-1 \\ d\left(p_{n}\left(X_{i}\right), p_{n}\left(V_{j}\right)\right) & 3 \cdot 2^{r-3} & \text { for } 1 \leq i \leq r-2, \\ & & 1 \leq j \leq r-2, \\ & & j \neq i, i+1 \\ d\left(p_{n}\left(V_{r}\right), p_{n}\left(X_{r-2}\right)\right) & 3 \cdot 2^{r-3} & \\ d\left(p_{n}\left(V_{0}\right), p_{n}\left(V_{i}\right)\right) & 3 \cdot 2^{r-3}-1 & \text { for } 2 \leq i \leq r-2 \\ d\left(p_{n}\left(X_{i}\right), p_{n}\left(V_{r-1}\right)\right) & 5 \cdot 2^{r-4} & \text { for } 1 \leq i \leq r-3, \\ d\left(p_{n}\left(V_{r}\right), p_{n}\left(X_{i}\right)\right) & 5 \cdot 2^{r-4} & \text { for } 1 \leq i \leq r-3 \\ d\left(p_{n}\left(X_{i}\right), p_{n}\left(X_{j}\right)\right) & 9 \cdot 2^{r-5} & \text { for } 1 \leq i \leq j-2 \leq r-5 \\ d\left(p_{n}\left(X_{i}\right), p_{n}\left(X_{r-2}\right)\right) & 2^{r-2} & \text { for } 1 \leq i \leq r-4\end{array}$

Table 3: Some distances of projections into $Q_{n}$, where $n=3 \cdot 2^{r-2}$

between the pairs of the form $\left\{p_{n}\left(X_{r-2}\right), p_{n}\left(X_{i}\right)\right\}$ where $1 \leq i \leq r-4$. Thus in $G_{p_{n}(U)}$ the only vertices of degree zero are $p_{n}\left(V_{0}\right)$ and $p_{n}\left(V_{r}\right)$, the only vertices of degree one are $p_{n}\left(V_{1}\right)$ and $p_{n}\left(V_{r-1}\right)$, and the vertices of degree two are precisely the vertices $p_{n}\left(V_{i}\right)$ where $2 \leq i \leq r-2$.

Recall that since $G_{p_{n}(U)}$ is defined by distances in $Q_{n}$, to prove that $p_{n}(G)$ is 1-distinguishable in $Q_{n}$ we can use information from $Q_{n}$ itself and information from $G_{p_{n}(U)}$. Notice that in both $V_{0}$ and $V_{r}$ the second $2^{r-1}$ coordinates are zeros. In particular, their second $2^{r-1}$ coordinates are the same. Thus distances involving $V_{0}$ and $V_{r}$ are reduced by the same amount in the projection to $Q_{n}$. Thus $p_{n}\left(V_{0}\right)$ still attains greater distances with vertices of $p_{n}(U)-\left\{p_{n}\left(V_{0}\right), p_{n}\left(V_{r}\right)\right\}$ than $p_{n}\left(V_{r}\right)$ can attain. Since automorphisms preserve distance, $p_{n}\left(V_{0}\right)$ and $p_{n}\left(V_{r}\right)$ cannot be transposed by any automorphism that preserves $p_{n}(U)$. (Note that this is true for any $2^{r-1}<n<2^{r}$; it will be used again in Case 2.) Thus we can distinguish $p_{n}\left(V_{0}\right)$ and $p_{n}\left(V_{r}\right)$ in $p_{n}(U)$.

If $p_{n}\left(V_{1}\right)$ and $p_{n}\left(V_{r-1}\right)$ have different distances from $p_{n}\left(V_{0}\right)$ then they are distinguished from each other. However, if $p_{n}\left(V_{1}\right)$ and $p_{n}\left(V_{r-1}\right)$ have the same distance from $p_{n}\left(V_{0}\right)$, we can replace $V_{0}$ (and $p_{n}\left(V_{0}\right)$ ) with the vertex of all zeros. This does not change any argument given so far. (The only possible concern is the distance between $p_{n}\left(V_{0}\right)$ and $p_{n}\left(V_{1}\right)$. With the change to $V_{0}$, when $n=2^{r-1}$ the distance between $p_{n}\left(V_{0}\right)$ and $p_{n}\left(V_{1}\right)$ drops to $2^{r-2}$, which gives $p_{n}\left(V_{0}\right)$ a neighbor in the distance relationship graph. However, since $V_{0}$ and $V_{1}$ differ in their $\left(2^{r-1}+1\right)^{s t}$ coordinate, when $n>2^{r-1}$ their distance is still greater than $2^{r-2}$.) The change in $V_{0}$ will decrease the distance between $p_{n}\left(V_{0}\right)$ and $p_{n}\left(V_{1}\right)$ by one and increase the distance between $p_{n}\left(V_{0}\right)$ and $p_{n}\left(V_{r-1}\right)$ by one, thereby distinguishing $p_{n}\left(V_{1}\right)$ and $p_{n}\left(V_{r-1}\right)$. Again, since the only requirement is that $n>2^{r-1}$, this argument will still be valid in Case 2.

The vertex $p_{n}\left(X_{1}\right)$ (resp. $p_{n}\left(X_{r-2}\right)$ ) is the only one adjacent to the vertex $p_{n}\left(V_{1}\right)$ (resp. $\left.p_{n}\left(V_{r-1}\right)\right)$ in $G_{p_{n}(U)}$. Thus $p_{n}\left(X_{1}\right)$ and $p_{n}\left(X_{r-2}\right)$ are distinguished. The vertex $p_{n}\left(V_{2}\right)$ (resp. $\left.p_{n}\left(V_{r-2}\right)\right)$ is the only vertex of degree 2 at distance 2 from $p_{n}\left(V_{1}\right)\left(\right.$ resp. $p_{n}\left(V_{r-1}\right)$ ) in $G_{p_{n}(U)}$. Thus these are also distinguished. 
The vertex $p_{n}\left(X_{2}\right)$ (resp. $\left.p_{n}\left(X_{r-3}\right)\right)$ is the only one adjacent to both $p_{n}\left(X_{1}\right)$ and $p_{n}\left(V_{2}\right)$ (resp. $p_{n}\left(X_{r-2}\right)$ ) and $p_{n}\left(V_{r-2}\right)$ ) in $G_{p_{n}(U)}$; thus they are distinguished. Continue in this manner to see that all vertices in $G_{p_{n}(U)}$ are distinguished. Thus $p_{n}(U)$ is a 1-distinguishable determining set.

Note that in the argument above we distinguished all of $p_{n}(U)-\left\{p_{n}\left(V_{r}\right)\right\}$ without using the fact that $p_{n}\left(V_{r}\right)$ was itself distinguished. Once $p_{n}\left(V_{0}\right)$ was distinguished, the remainder of $p_{n}(U)-\left\{p_{n}\left(V_{0}\right), p_{n}\left(V_{r}\right)\right\}$ could be distinguished. This results in the distinguishing of $p_{n}\left(V_{r}\right)$ by elimination. This argument will be used again in Case 2 below.

Case 2: $2^{r-1}<n<3 \cdot 2^{r-2}$.

When $2^{r-1}<n<3 \cdot 2^{r-2}$ define the distance relationship graph $G_{p_{n}(U)}$ on $p_{n}(U)$ so that there is an edge between a pair of vertices when their distance is strictly less than $2^{r-2}$. Recall that the distances between related pairs have dropped strictly below $2^{r-2}$ since $n<3 \cdot 2^{r-2}$. Table 2 indicates that the distance between a pair of the form $\left\{p_{2^{r-1}}\left(V_{0}\right), p_{2^{r-1}}\left(V_{i}\right)\right\}$ for $2 \leq i \leq r-2$ is $2^{r-2}-1$ in $Q_{2^{r-1}}$. However, $V_{0}$ and $V_{i}$ differ in their $\left(2^{r-1}+1\right)^{s t}$ coordinate. Thus the distance between their projections in $Q_{n}$ is at least $2^{r-2}$. From Table 2 we see that the only other non-related pairs of vertices that might have distance smaller than $2^{r-2}$ in $Q_{n}$ are the pairs $\left\{p_{n}\left(X_{i}\right), p_{n}\left(X_{j}\right)\right\}$ where $1 \leq i \leq j-2 \leq r-4$ and the pairs $\left\{p_{n}\left(V_{r}\right), p_{n}\left(X_{i}\right)\right\}$ where $1 \leq i \leq r-2$. Thus we see that in $G_{p_{n}(U)}, p_{n}\left(V_{0}\right)$ still has degree zero, $p_{n}\left(V_{1}\right)$ and $p_{n}\left(V_{r-1}\right)$ still have degree one, and $p_{n}\left(V_{i}\right)$ with $2 \leq i \leq r-2$ still have degree two. Further $p_{n}\left(X_{i}\right)$ with $1 \leq i \leq r-2$ still have degree greater than two. However, the degree of $p_{n}\left(V_{r}\right)$ varies depending on the value of $n$.

If the degree of $p_{n}\left(V_{r}\right)$ in $G_{p_{n}(U)}$ is greater than zero, then $p_{n}\left(V_{0}\right)$ is the only vertex of degree zero and is thus distinguished.

Suppose the degree of $p_{n}\left(V_{r}\right)$ in $G_{p_{n}(U)}$ is zero. Then $p_{n}\left(V_{0}\right)$ and $p_{n}\left(V_{r}\right)$ are the only vertices of degree zero in $G_{p_{n}(U)}$. Thus any automorphism that preserves $p_{n}(U)$ either fixes them both or transposes them. However, the argument given in Case 1 shows that when $2^{r-1}<n<2^{r}$, we can distinguish $p_{n}\left(V_{0}\right)$ and $p_{n}\left(V_{r}\right)$ by the distances they attain.

Thus in either case, $p_{n}\left(V_{0}\right)$ is distinguished and we can use the arguments of Case 1 to distinguish the remaining vertices of $p_{n}(U)$.

Thus we have found a 1-distinguishable determining set, i.e. a distinguishing class, of size $2 r-1=2\left\lceil\log _{2} n\right\rceil-1$ for $Q_{n}$.

Corollary 7. For $n \geq 5,\left\lceil\log _{2} n\right\rceil+1 \leq \rho\left(Q_{n}\right) \leq 2\left\lceil\log _{2} n\right\rceil-1$.

Proof. By the remarks following Theorem 2, every distinguishing class for $Q_{n}, n \geq 4$, is also a determining set. By [5] a smallest such set for $Q_{n}$ has size $\left\lceil\log _{2} n\right\rceil+1$. This provides the lower bound.

The only 2-distinguishable case for $Q_{n}$ that is not covered in Theorem 6 is $Q_{4}$. The proof technique fails in this case because the given set $U$ has no $X_{i}$ when $n<5$. If the results of Theorem 6 held for $Q_{4}$, it would have a distinguishing class of size three. Since three is also the determining number for $Q_{n}$, such a distinguishing class would also be a minimum size determining set. It is not hard to show that there is a single isomorphism class of minimum size determining sets for $Q_{4}$, and that one of its members, $U=\{0000,1010,1100\}$, has a 
nontrivial set stabilizer. Thus every minimum size determining set for $Q_{4}$ has a nontrivial stabilizer and therefore cannot be a distinguishing class.

Thus the result of Theorem 6 does not hold for $n<5$.

\section{Open Questions}

Question 8. For $n \geq 5$ is there a distinguishing class for $Q_{n}$ that is smaller than $2\left\lceil\log _{2} n\right\rceil-$ 1 ?

Question 9. For $n \geq 5$ we saw that $\rho\left(Q_{n}\right)$ is no bigger than a constant multiple of $\operatorname{Det}\left(Q_{n}\right)$. That is, $\rho\left(Q_{n}\right)=O\left(\operatorname{Det}\left(Q_{n}\right)\right)$ in this case. For what other infinite families of graphs is this true?

\section{Acknowledgments}

The author thanks Wilfried Imrich for useful conversations about determining sets and distinguishing labelings of Cartesian products. The author also thanks the referees for finding a computational error in the original proof of Theorem 6 and for a careful reading of the manuscript.

\section{References}

[1] M. O. Albertson and D. L. Boutin, Using determining sets to distinguish Kneser graphs, Electron. J. Combin. 14 (2007), \#R20.

[2] M. O. Albertson and K. L. Collins, Symmetry breaking in graphs, Electron. J. Combin. 3 (1996), \#R18.

[3] B. Bogstad and L. J. Cowen, The distinguishing number of the hypercube, Discrete Math. 283 (2004), 29-35.

[4] D. L. Boutin, Identifying graph automorphisms using determining sets, Electron. J. Combin. 13 (2006), \#R78.

[5] D. L. Boutin, The determining number of Cartesian products, J. Graph Theory, forthcoming.

[6] T. Fukuda, S. Negami and T. Tucker, 3-connected planar graphs are 2-distinguishable with few exceptions, preprint, 2006.

[7] W. Imrich, personal communication.

[8] W. Imrich and S. Klavžar, Distinguishing Cartesian powers of graphs, J. Graph Theory 53 (2006), 250-260.

[9] W. Imrich and S. Klavžar, Product graphs, Wiley-Interscience Series in Discrete Mathematics and Optimization, Wiley-Interscience, New York, 2000. 